\title{
Miniaturisation of photoacoustic sensing systems using MEMS transducer arrays and MEMS scanning mirrors
}

\author{
Jonas Kusch, Gordon M.H. Flockhart, Ralf Bauer, Deepak Uttamchandani \\ Centre for Microsystems \& Photonics, Department of Electronic \& Electrical Engineering \\ University of Strathclyde \\ Glasgow, UK \\ jonas.kusch@strath.ac.uk
}

\begin{abstract}
A custom-built thin film aluminium nitride MEMS ultrasound transducer and its application to photoacoustic sensing is presented. The photoacoustic signal is generated using a commercial Nd:YAG laser and, for the first time, using a high pulse repetition rate MEMS Q-switched Nd:YAG laser.
\end{abstract}

Keywords—ultrasound, MEMS, transducer, photoacoustics

\section{INTRODUCTION}

Photoacoustic (PA) sensing offers the potential for improvements in both specificity and resolution when compared to conventional ultrasound as a sensing technique in areas ranging from biomedical imaging [1] to non-destructive evaluation [2]. This has caused PA sensing to attract a lot of attention especially in biomedical applications. Driving towards miniaturised PA sensing systems, a variety of miniaturised ultrasound transducers are available, such as capacitive micromachined ultrasonic transducers (cMUT) and micro ring resonators (MRR). cMUTs consist of a thin film - released by etching a cavity into a silicon substrate - onto which metal is deposited. The capacitor created in this manner changes its capacitance due to pressure induced deflection of the membrane [3]. MRRs consist of an optical micro ring resonator which is deformed by incoming pressure waves and thus its optical resonance modes are altered [4]. These transducer types offer broad bandwidths but require to be actively interrogated by either a high voltage of typically greater than $70 \mathrm{~V}$ (cMUT) or narrow-band laser light (MRR). Piezoelectric ultrasound transducers, on the other hand, offer a way of passively detecting pressure changes by generating a voltage due to pressure wave induced deformations. Furthermore, such passive piezoelectric transducers can be miniaturised using MEMS fabrication techniques, leading the way towards miniaturised PA sensing systems.

PA sensing systems can be further miniaturised by replacing conventional solid state lasers with high-power pulsed laser diodes (PLD) which offer pulse repetition frequencies (PRF) of up to $7 \mathrm{kHz}$ compared to $10 \mathrm{~Hz}$ for solid state lasers (e.g. Nd:YAG) but also have increased pulse widths of $>120 \mathrm{~ns}$ compared to $<10 \mathrm{~ns}$ for solid state lasers [5], [6]. Recently, PLD with pulse width of $\tau<10$ ns became commercially available for wavelengths $\lambda<640 \mathrm{~nm}$. For biomedical imaging wavelengths of $650 \mathrm{~nm}<\lambda<1350 \mathrm{~nm}$ are of importance as attenuation is decreased by a factor of 100 [7]. PLD were experimentally shown to work at pulse energies of $<$ $220 \mathrm{~nJ}$ for $\lambda<840 \mathrm{~nm}$ at high PRF (f $=625 \mathrm{kHz}$ ) and short pulse widths $(\tau<10 \mathrm{~ns})$ [8]. An alternative to PLDs, to achieve higher pulse energies, is to use an active Q-switched Nd:YAG laser with an intra-cavity MEMS resonant mirror as active element. This has been shown to yield pulse widths down to 38 ns at PRF of $17.6 \mathrm{kHz}$ [9] and pulse energies of above $40 \mu \mathrm{J}$ when the MEMS resonant mirrors were dielectric-coated [10].

In this work we present the application of a customdesigned and fabricated piezoelectric MEMS ultrasound sensor to detect photoacoustic signals. Furthermore, we report for the first time on the generation of photoacoustic signals using a MEMS based Q-switched Nd:YAG laser.

\section{DESIGN AND FABRICATION}

A piezoelectric MEMS transducer array with identical elements and uniform spacing was designed with an intended operating frequency in the low $\mathrm{MHz}$ range, as this allows for greater sensing depths of photoacoustic signals in tissue. Sensing depths of $>5 \mathrm{~cm}$ have been reported using $2.25 \mathrm{MHz}$ transducers [11]. Our disc-shaped MEMS transducer designs were modelled in water (as an approximation for biological tissue) using the pressure acoustics module of COMSOL Multiphysics (COMSOL Inc). They showed a first resonance frequency at $1.18 \mathrm{MHz}$. This array was designed to enable changes in the overall sensing area of the chip by combining different numbers of transducer elements (see Fig. 1 a). Furthermore, this enables such transducers to have future applications in an acoustic phased array setup. Our designs were fabricated using PiezoMUMPs (MEMSCAP Inc.) - a
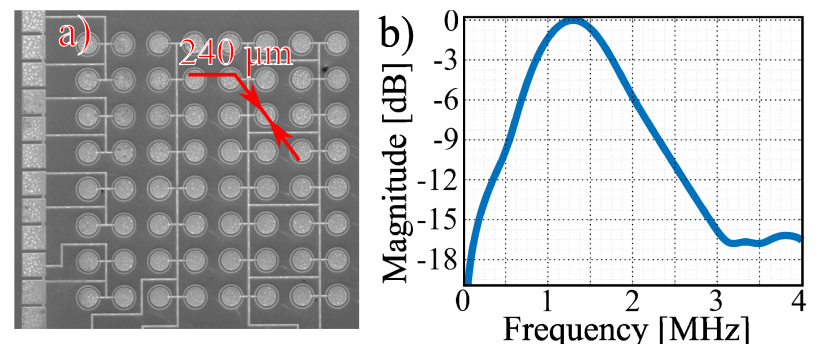

Fig 1: a) SEM image of the AlN thin-film disc-shaped ultrasound transducer with a diameter of $240 \mu \mathrm{m}$. The array consists of 64 individual elements with a diameter of $240 \mu \mathrm{m}$ grouped together in different sized sub-arrays. b) The transducer has a centre frequency of $1.3 \mathrm{MHz}$ and a $-6 \mathrm{~dB}$ bandwidth of $1.29 \mathrm{MHz}$ in oil as calculated from the transducer response to photoacoustic excitation. 
commercial cost-efficient multi-user process for the production of piezoelectric MEMS devices on silicon-on-insulator (SOI) substrate The transducer elements are nominally made of a 500 $\mathrm{nm}$ aluminium nitride piezoelectric layer covered by a $1 \mu \mathrm{m}$ thick aluminium layer acting as top electrode on a $10 \mu \mathrm{m}$ silicon-on-insulator device layer. This is fully backside released from a $400 \mu \mathrm{m}$ silicon substrate wafer. Our fabricated transducer elements had a diameter of $240 \mu \mathrm{m}$ (see Fig. 1 a) and a pitch of $400 \mu \mathrm{m}$.

\section{MEMS Q-SWITCHED ND:YAG}

A MEMS mirror and a flat partially reflective mirror $(\mathrm{R}=$ $80 \%$ ) create an optical cavity around a commercial diode sidepumped Nd:YAG crystal (RBA30-1C2 Resonator Module, Northrop Grumman) (see Fig 2 a). The SOI MEMS resonant scanning mirror has a $700 \mu \mathrm{m}$ mirror diameter with a $250 \mathrm{~nm}$ thick gold coating, showing a concave surface curvature with a radius of curvature (ROC) of $400 \mathrm{~mm}$. It is clamped to the substrate by two rectangular beams and actuated by an electrostatic comb-drive driven with a square input voltage with a peak-to-peak voltage of $\mathrm{V}_{\mathrm{pp}}=200 \mathrm{~V}$ at a frequency of $11.9 \mathrm{kHz}$. The comb-drive is made of ten interleaving $160 \mu \mathrm{m}$ long silicon bars spaced $6 \mu \mathrm{m}$ apart [9]. This set-up gives laser pulses with an average power of up to $55 \mathrm{~mW}$, PRF of 11.9 $\mathrm{kHz}$ and pulse widths of $50 \mathrm{~ns}$ (see Fig 3). Furthermore, no double pulses were observed. Thus, its PRF is higher by a factor of 1000 compared to commercial Nd:YAG lasers with usual PRF around $10 \mathrm{~Hz}$, and its pulse energies is higher by a factor of 20 compared to short pulse width PLD.

\section{METHODS \& RESUlTS}

\section{A. Photoacoustic characterisation}

To measure its acoustic response, the MEMS transducer was characterised using photoacoustic excitation [12]. Here a commercial Nd:YAG laser (Brilliant B, Quantel), frequency doubled at $532 \mathrm{~nm}$ with $10 \mathrm{~ns}$ pulses, a pulse repetition frequency of $10 \mathrm{~Hz}$, pulse energies of $2.2 \mathrm{~mJ}$ is focussed to a focal spot size of $250 \mu \mathrm{m}$ using a $35 \mathrm{~mm}$ lens onto an $8 \%$ $(\mathrm{m} / \mathrm{m})$ gelatine phantom. The phantom consisted of a clear layer and a highly-absorbing layer to which $0.4 \%(\mathrm{~m} / \mathrm{m})$ India ink was added. This phantom and the MEMS transducer were immersed in an oil bath for acoustic coupling (see Fig. 2 b). The transducer output is amplified by $+40 \mathrm{~dB}$ (DHPVA-200, Femto) and the waveforms recorded using a $1.5 \mathrm{GHz}$ oscilloscope (Agilent, Infiniium). The transducer response was averaged over 256 samples and recorded for PA signals in the absorbing layer. The centre frequency and bandwidth were derived from the signal's power spectral density (PSD) by application of the fast Fourier transform. This yielded a centre frequency of $1.3 \mathrm{MHz}$ and $-6 \mathrm{~dB}$ bandwidth of $1.29 \mathrm{MHz}$ (see Fig. 1 b).

\section{B. Photoacoustic signal generation with commercial Nd:YAG}

The MEMS transducer's performance for different pulse energies was tested using the setup in part IV (A). Here, the pulse energy was decremented using neutral density filters (NDK01, Thorlabs) from $2.1 \mathrm{~mJ}$ to $0.66 \mu \mathrm{J}$. The laser pulses

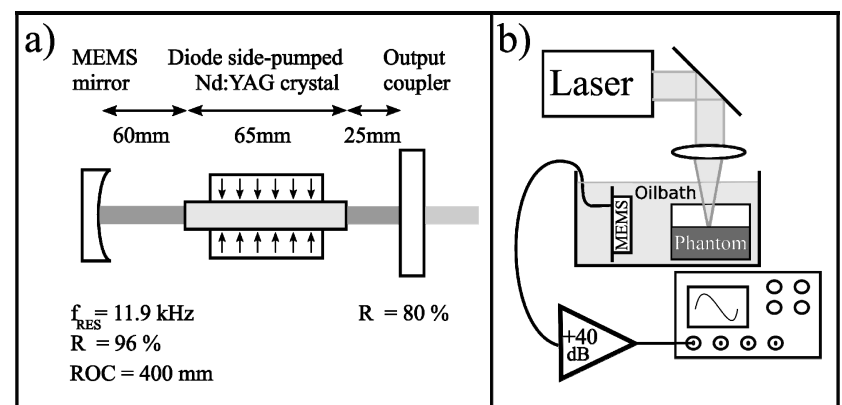

Fig 2: a) The MEMS mirror, a diode side-pumped Nd:YAG crystal and a curved partially reflective mirror form a laser cavity. This cavity is actively Q-switched by the MEMS mirror moving in and out of alignment. b) The experimental setup for photoacoustic sensing consists of a laser focussed onto a sample by a lens. The generated photoacoustic signal is detected by the MEMS ultrasound transducer, amplified by $+40 \mathrm{~dB}$ and then recorded on an oscilloscope.

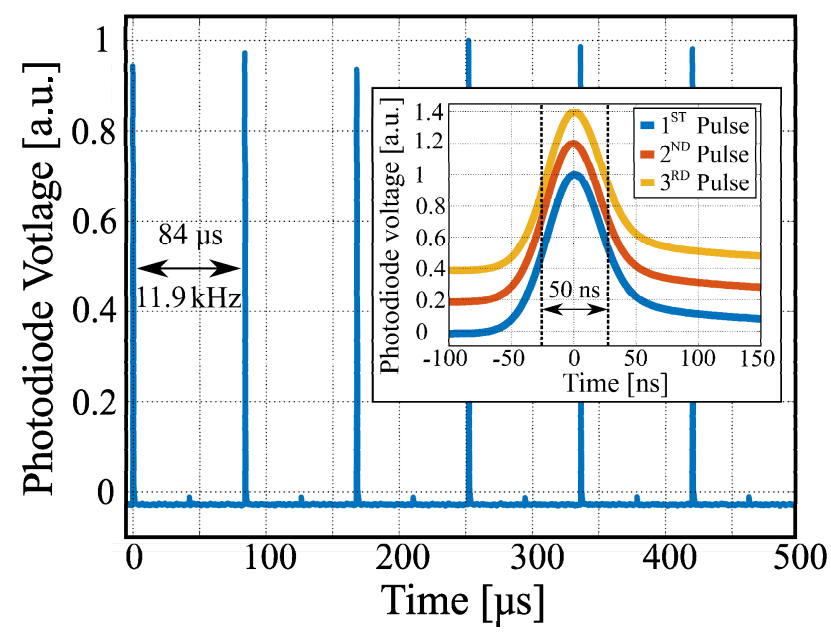

Fig 3: The MEMS Q-switched laser generates laser pulses with an average power of $55 \mathrm{~mW}$ at a PRF of $11.9 \mathrm{kHz}$. The inset shows the first three pulses offset by 0.2 a.u. with pulse widths of $50 \mathrm{~ns}$.

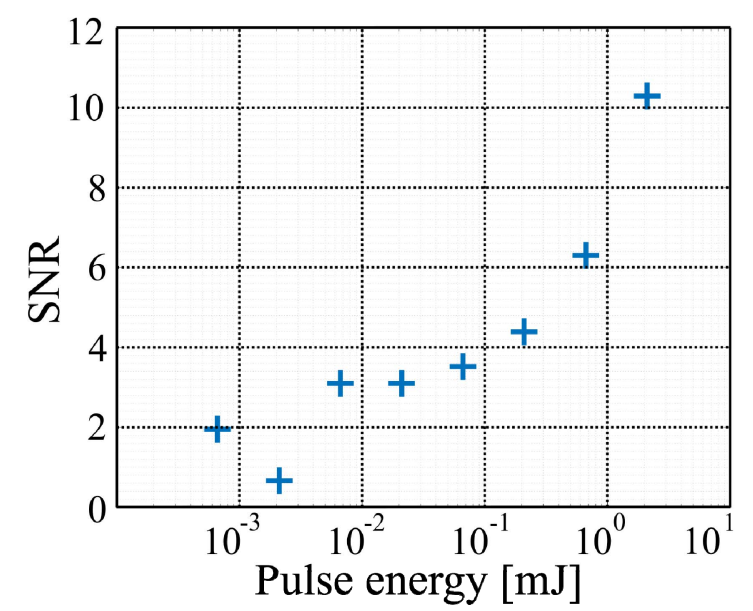

Fig 4: The SNR increases exponentially for pulse energies greater than $5 \mu \mathrm{J}$. The PA signal were generated by the commercial Nd:YAG (Brilliant B). 
were incident onto the broadband optical absorbing gelatine phantom and the generated PA waves detected using the MEMS transducer array. The recorded waveforms were the average of 256 captured waveforms. The PA signal in the recorded waveforms was then windowed using a Hanning window and subjected to the FFT. The signal power was calculated by integrating over the PSD. The noise power was calculated in a similar manner by windowing over the noise. The signal-to-noise ratio (SNR) was then calculated as the ratio of the signal power and the noise power. The SNR increases exponentially with pulse energy for pulse energies greater than $3 \mu \mathrm{J}$ (see Fig. 4).

\section{Photoacoustic signal generation with MEMS Q-switched Nd:YAG}

The MEMS Q-switched Nd:YAG laser at $1064 \mathrm{~nm}$ with 50 ns pulses, a pulse repetition frequency of $11.9 \mathrm{kHz}$ and pulse energies of $4.42 \mu \mathrm{J}$ focussed onto the gelatine phantom using a lens with a focal length of $30 \mathrm{~mm}$ was used to generate PA signals. The transducer's response was averaged using 256 samples and recorded with the laser beam hitting the gelatine phantom (see Fig. 5, blue solid line) and with the laser beam blocked (see Fig. 5, red dotted line). In the former, a clear photoacoustic response with a $\mathrm{V}_{\mathrm{pp}}=8.1 \mathrm{mV}$ can be observed and in the latter the noise floor has a peak-to-peak voltage of $\mathrm{V}_{\mathrm{pp}}=1.8 \mathrm{mV}$. The SNR (V/V) of the detected signal was 4.6 .

\section{DISCUSSION AND CONCLUSION}

The custom-built MEMS ultrasound transducer array using disc shaped $240 \mu \mathrm{m}$ aluminium nitride thin film elements was measured to have a centre frequency of $1.3 \mathrm{MHz}$ and a $-6 \mathrm{~dB}$ bandwidth of $1.29 \mathrm{MHz}$. For the first time it was shown that a MEMS Q-switched Nd:YAG laser with high PRF of $>11 \mathrm{kHz}$ can be used to generate photoacoustic signals. These lasers have an advantage over high-power PLDs as their pulse repetition rates are higher and their pulse widths are narrower. Furthermore, their significant advantage over the short pulse width PLD is that their pulse energy is higher by a factor of 20 . This can lead to faster imaging and sensing interrogation requiring smaller averaging sizes for noise compensation.

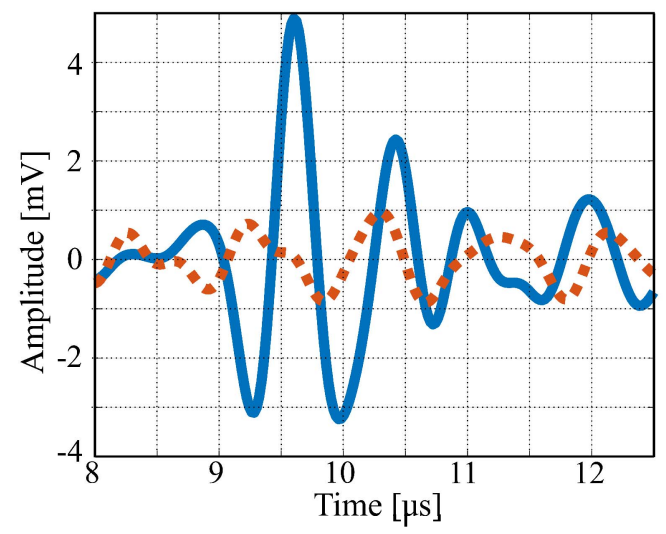

Fig 5: A photoacoustic pulse (blue solid line) generated by the MEMS Q-switched Nd:YAG laser. The SNR is 4.6. It was calculated from the pulse and the noise background (red dotted line) which was recorded with the laser beam blocked.

\section{REFERENCES}

[1] P. Beard, "Biomedical photoacoustic imaging," Interface Focus, vol. 1, no. June, pp. 602-631, 2011.

[2] H. Huan, A. Mandelis, B. Lashkari, and L. Liu, "Frequency-Domain Laser Ultrasound ( FDLU ) Nondestructive Evaluation of Stress - Strain Behavior in an Aluminum Alloy," Int. J. Thermophys., vol. 38, no. 4, pp. 1-11, 2017.

[3] S. H. Pun, Y. Yu, J. Zhang, J. Wang, C. H. Cheng, K. F. Lei, Z. Yuan, and P. U. Mak, "Monolithic Multiband CMUTs for Photoacoustic Computed Tomography with In Vivo Biological Tissue Imaging," IEEE Trans. Ultrason. Ferroelectr. Freq. Control, vol. 65, no. 3, pp. 465-475, 2018.

[4] H. Li, B. Dong, Z. Zhang, H. F. Zhang, and C. Sun, "A transparent broadband ultrasonic detector based on an optical micro-ring resonator for photoacoustic microscopy," Sci. Rep., vol. 4, 2014.

[5] T. Wang, S. Nandy, H. S. Salehi, P. D. Kumavor, and Q. Zhu, "A low-cost photoacoustic microscopy system with a laser diode excitation," Biomed. Opt. Express, vol. 5, no. 9, pp. 20097-20102, 2014.

[6] P. K. Upputuri and M. Pramanik, "Pulsed laser diode based optoacoustic imaging of biological tissues," Biomed. Phys. Eng. Express, vol. 1, 2015.

[7] A. M. Smith, M. C. Mancini, and S. Nie, "Second window for in vivo imaging," Nat Nanotechnol., vol. 4, no. 11, pp. 710-711, 2009.

[8] A. Stylogiannis, L. Prade, A. Buehler, J. Aguirre, G. Sergiadis, and V. Ntziachristos, "Continuous wave laser diodes enable fast optoacoustic imaging," Photoacoustics, vol. 9, pp. 31-38, 2018.

[9] R. Bauer, W. Lubeigt, and D. Uttamchandani, "Qswitching of Nd: YAG solid-state laser with intracavity MEMS resonant scanning mirror," IEEE Xplore 2012 Int. Conf. Opt. MEMS Nanophotonics, vol. 10, pp. 81-82, 2012.

[10] R. Bauer, A. Paterson, C. Clark, D. Uttamchandani, S. Member, and W. Lubeigt, "Output Characteristics of Q-switched Solid-State Lasers Using Intracavity MEMS Micromirrors," IEEE J. Sel. Top. QUANTUM Electron., vol. 21, no. 1, 2015.

[11] G. $\mathrm{Ku}$ and L. V. Wang, "Deeply penetrating photoacoustic tomography in biological tissues enhanced with an optical contrast agent," Opt. Lett., vol. 30 , no. 5 , p. $507,2005$.

[12] J. Kusch, G. M. H. Flockhart, R. Bauer, and D. Uttamchandani, "Photoacoustic characterization of custom-made thin film AIN MEMS ultrasound transducers," IEEE Xplore 2018 Int. Conf. Opt. MEMS Nanophotonics, 2018. 